\title{
NEW INEQUALITIES FOR G-FRAMES IN HILBERT $C^{*}$-MODULES
}

\author{
ZHONG-QI XIANG
}

Abstract. In this paper, we establish several new inequalities for $\mathrm{g}$-frames in Hilbert $C^{*}$-modules which are different in structure from those previously obtained by Balan et al. for Hilbert space frames. We also present some equalities and inequalities for $\mathrm{g}$-frames in Hilbert $C^{*}$-modules with Moore-Penrose inverses and show that they are more general and cover some results in [Xiao, XC, Zeng, XM: Some properties of g-frames in Hilbert $C^{*}$-modules. J. Math. Anal. Appl. 363 (2), 399-408 (2010)].

Mathematics subject classification (2010): 46L99, 42C15, 46H25.

Keywords and phrases: Hilbert $C^{*}$-module, frame, inequality, Moore-Penrose inverse.

\section{REFERENCES}

[1] R. J. Duffin, A. C. Schaeffer, A class of nonharmonic Fourier series, Trans. Amer. Math. Soc. 72, 2 (1952), 341-366.

[2] I. Daubechies, A. Grossmann, Y. Meyer, Painless nonorthogonal expansions, J. Math. Phys. 27, 5 (1986), 1271-1283.

[3] P. CASAzZA, The art of frame theory, Taiwanese J. Math. 4, 2 (2000), 129-201.

[4] O. Christensen, An Introduction to Frames and Riesz Bases, Birkhäuser, Boston (2003).

[5] W. C. Sun, G-frames and g-Riesz bases, J. Math. Anal. Appl. 322, 1 (2006), 437-452.

[6] P. CAsazZa, G. Kutyniok, Frames of subspaces, In Wavelets, Frames and Operator Theory. Contemp. Math., Vol. 345, pp. 87-113. Amer. Math. Soc., Providence (2004).

[7] O. Christensen, Y. C. Eldar, Oblique dual frames and shift-invariant spaces, Appl. Comput. Harmon. Anal. 17, 1 (2004), 48-68.

[8] S. Li, H. OGawA, Pseudoframes for subspaces with applications, J. Fourier Anal. Appl. 10, 4 (2004), 409-431.

[9] A. Aldroubi, C. Cabrelli, U. Molter, Wavelets on irregular grids with arbitrary dilation matrices and frame atomics for $L^{2}\left(\mathbb{R}^{d}\right)$, Appl. Comput. Harmon. Anal. 17, 2 (2004), 119-140.

[10] M. FrAnK, D. R. LARSON, Frames in Hilbert $C^{*}$-modules and $C^{*}$-algebras, J. Operator Theory 48 , 2 (2002), 273-314.

[11] A. Khosravi, B. Khosravi, Fusion frames and $g$-frames in Hilbert $C^{*}$-modules, Int. J. Wavelets Multiresolut. Inf. Process. 6, 3 (2008), 433-446.

[12] X. C. XiAo, X. M. Zeng, Some properties of g-frames in Hilbert $C^{*}$-modules, J. Math. Anal. Appl. 363, 2 (2010), 399-408.

[13] A. Alijani, M. A. Dehghan, G-frames and their duals for Hilbert $C^{*}$-modules, Bull. Iranian Math. Soc. 38, 3 (2012), 567-580.

[14] A. Askarizadeh, M. A. Dehghan, G-frames as special frames, Turkish J. Math. 37, 1 (2013), $60-70$.

[15] A. Alijani, Generalized Frames with $C^{*}$-Valued Bounds and their Operator Duals, Filomat, 29, 7 (2015), 1469-1479.

[16] R. Balan,, P. Casazza, D. Edidin, G. Kutyniok, A new identity for Parseval frames, Proc. Amer. Math. Soc. 135, 4 (2007), 1007-1015.

[17] P. GĂVRUŢA, On some identities and inequalities for frames in Hilbert spaces, J. Math. Anal. Appl. 321, 1 (2006), 469-478. 
[18] Q. X. XU, L. J. Sheng, Positive semi-definite matrices of adjointable operators on Hilbert $C^{*}$ modules, Linear Algebra Appl. 428, 4 (2008), 992-1000. 